Plenary 1:

Enhancing inclusivity and diversity into the new norm: what can we do better in medical education?

\title{
'Think global, act local'-ensuring inclusivity and diversity of medical education in the new norm
}

\begin{abstract}
Yusoff, M.S.B.
Malaysia

The new norm, due to the recent pandemic, is inevitable and has changed the way people live. It leads to social distancing, personalized space, small group-based activities, and increase the use of digital platforms in daily activity. It has also given a great impact on the way medical education being carried out around the globe. The main challenge is to ensure its inclusivity and diversity remain intact and hopefully not worsening. Inclusivity is about equal access to opportunities and resources for individuals who might otherwise be excluded or marginalized, while diversity is about understanding that each individual is unique and recognizing individual differences. Having several months of experience in delivering medical education through digital platforms has made us realize the need to sustain or do better to ensure the inclusivity and diversity of medical education in the new norm. There are many challenges faced by us to provide the best quality of medical education to learners. 'Think Global, Act Local' is a concept that considers enablers and barriers in the local setting, and charting strategies based on the best practices around the globe. 'Think Global, Act Local' can be a way to ensure medical education becoming more inclusive and more prepared to embrace diversity in the new norm.
\end{abstract}

